\title{
Review on Psyllium Husk (Plantago ovata): A Novel Superfood for Human Health
}

\author{
S. D. Katke ${ }^{1 *}$, H. W. Deshpande ${ }^{1}$ and A. R. Tapre ${ }^{2}$ \\ ${ }^{1}$ Department of Food Microbiology \& Safety, College of Food Technology, \\ VNMKV, Parbhani, Maharashtra, India \\ ${ }^{2}$ Department of Food Processing Technology, A.D. Patel Institute of Technology, \\ Anand, Gujarat, India \\ *Corresponding author
}

\section{A B S T R A C T}

K e y w o r d s
Psyllium husk,
Constipation,
Diabetes,
Cholesterol,
Plantago ovata,
Functional food,
Isabgol, Isapghula

\section{Keywords}

Psyllium husk,

Constipation

Isabgol, Isapghula

\section{Introduction}

Plantago ovata commonly known as 'Psyllium' in English and 'Isabgol' in Hindi belongs to the family of Plantaginaceae, is a $10-45 \mathrm{~cm}$ short-stemmed annual herb known by different names such as ashwagolam, aspaghol, aspagol, bazarqutuna, blond Psyllium. Isabgol has high fiber content and acts like a sponge serving to clean the bowels and is extensively cultivated in many parts of

\begin{abstract}
Commonly called Psyllium (Ispaghula Psyllium, Plantago ispaghula and Plantago ovata), has a long history of use as a dietary fiber supplement. Humankind has used Psyllium since ancient times; it has miraculous medicinal properties and health benefits that have been attributed to it throughout the ages. There are various health benefits of consuming psyllium. Its positive effects include relieving the symptoms of constipation as well as mild diarrhea. It improves digestion and cleanse colon, lower hypercholesterolemia, reduces the chances of heart diseases, helps in weight reduction, controls type- 2 diabetes, treats inflammatory bowel disease (ulcerative colitis) and hemorrhoids. In older days it was also applied topically to treat skin problems and insect stings. In food and dairy ustry, it is used as a thickening agent in the preparation of several food products. It has been popularly used as therapeutic agent. Dietary fibers from psyllium husk have been used extensively both as pharmacological supplements and food ingredients in processed food. The intent of this review is to summarize the functional benefits of Psyllium fiber consumption and to explore the potential application this fiber has for first-line dietary prevention of these diseases and disorders.
\end{abstract}

the globe. It is commercially an important Rabi season crop known for its medicinal properties. Apart from its husk (The seed coat is known as "husk") it is also being used in food industry especially in ice creams, biscuits and candies. The crop is mainly cultivated in the states of Rajasthan, Gujarat, Haryana and Madhya Pradesh.

Notably, India ranks first in Isabgol production (98\%) and is the sole supplier of 
seeds and husk in the international market. Among medicinal plants, Isabgol is the first ranked foreign exchange earner for the country (Rs.30 million annually). It contains a significant amount of proteins and husk yields colloidal mucilage which are valued for medicinal application and is used in Ayurveda, unani and allopathic systems of medicines. India is the largest producer and the main supplier of seed and husk to the world market. USA is the chief importer of Isabgol seeds and husk.

The crop has a large export demand in USA and Western Europe and about $90 \%$ of the production is exported to these countries. Also, the growing of this crop in winter season will not affect the production of succeeding monsoon crop and thus fits well in the cropping system. The seed husk finds variety of industrial applications. It is the main constituent of a number of laxative preparations containing sodium bicarbonate and various flavor's used in modern medicine.

\section{Global Scenario of Psyllium Husk Market}

India leads the global production as well as is the number one exporter in the world. India is the world leader in Psyllium in term of production as well as exports. The largest buyer of Isabgol from India is the United States, accounting for around $75 \%$ of the total husk exports from India. Germany is the largest single importer of seed. Psyllium research and field trials in the U.S. have been conducted mainly in Arizona and also in Washington. In India, Gujarat contributes $35 \%$ of world production of Psyllium Husk. In India Gujarat and Rajasthan states are the major producer states of psyllium. Psyllium husk is obtained from genus Plantago.

The husk is commercially used for mucilage production used in laxatives and other application of the same. The psyllium plant is native to South East Asia with India dominating the production by volume globally. The psyllium is high in soluble fiber content with detoxing effect over digestive system makes it a very apt nutraceutical. Psyllium husk is available in powder and as the whole husk. Psyllium husk dust is produced during the production psyllium husk powder which has application in construction and animal feed industry. Psyllium husk market is expected to be dominated by the North America region owing to its large laxative drug and supplement market. The natural origin of the psyllium husk and offering in organic is anticipated to drive the market over the forecast period.

The psyllium husk market is segmented on the basis of origin, offering, and application. On the basis of origin, the psyllium husk market can be segmented into conventional and organic. The organic segment to witness high CAGR over the forecast period owing to the global trend of organic and natural based supplements and nutraceuticals. The conventional segment is expected dominate in terms of volume share owing to costeffectiveness and low residue presence in the final product.

On the basis of offering, the psyllium husk market can be segmented into whole, powder, and dust. The whole segment is expected to dominate the global psyllium market in terms of volume share. The powder segment is anticipated to see strong CAGR over the forecast period owing to its increased application in supplement and pharmaceutical market. On the basis of application, the psyllium husk market can be segmented into dietary supplements, pharmaceutical, animal feed, and others. The dietary supplement segment is anticipated to dominate the global psyllium husk market owing to its high demand in the sector. 


\section{Psyllium products \& its application}

\section{Psyllium seeds}

Psyllium seeds are an Agri-farm product of the dried ripe seeds of Plantago ovata forks (farm. Plantaginaceae) and it has been cleaned free of all dust, Agri farm fibers, wastes, mud, stones and iron particles. The seed itself is made up of $40 \%$ Linoleic Acid (LA), an important fatty acid essential to health. Psyllium seed typically Light brown to moderate brown color and having faint odor. It contains about $70 \%$ soluble fiber and $30 \%$ insoluble fiber. It is made up of $40 \%$ Linoleic Acid (LA), an important fatty acid essential to health, $19 \%$ fiber content, $18.8 \%$ proteins, and $10-20 \%$ triglycerides. The seed mucilage consists of polysaccharides, which is a soluble fiber.

\section{Psyllium husk}

The husk of Psyllium is the actual coating of mucilage around the seed. It is considered pure dietary fiber and are the only part of the plant used in manufacturing Psyllium products. Its nutritional value consists mainly of glycosides, proteins, polysaccharides, vitamin B1, and choline. Fiber content of over $80 \%$ compared to less than $15 \%$ for oat bran and $10 \%$ for bran. The husk is composed mostly of a fiber called hemicellulose which is a complex carbohydrate found in whole grains, fruits and vegetables. Hemicellulose is indigestible; however, it is partially broken down in the colon and feeds the friendly intestinal flora. Psyllium seeds are processed to remove the outer coating of the seed to get the husk. Psyllium husk contains about 70\% soluble fiber and $30 \%$ insoluble fiber. This white fibrous material is used in Pharmaceutical, cosmetics and food product industries. Psyllium husk is available in various grades according to the purity and mesh size etc. to match the individual user needs. It is available mostly in four grades of purity: 99\% Pure, 98\% Pure, 95\% Pure and 85\% Pure. Psyllium husk and psyllium seeds are graded according to the purity and quality of the material (Fig. 1).

\section{Psyllium husk powder}

Psyllium husk powder is proceeded from the husk using pulverize machine using various particle mesh size. It is available mostly in four grades of purity: 99\% Pure, 98\% Pure, 95\% Pure and $85 \%$ Pure.

\section{Psyllium as a functional food}

Psyllium is most frequently added to breakfast cereals, meal replacements, bread, biscuits and other bakery products to improve the fiber content of the food but is also added to juices, shakes, yogurt, soups and even ice creams. It can also be used as a thickener in drinks or frozen desserts. Although Psyllium fiber has positive physiologic benefits, its high viscosity could make it difficult to incorporate into food products and a task is to get it acceptable to consumers. There are various commercial uses of Psyllium in food, pharmaceutical and other industries. Although obtaining dietary fiber from whole foods is preferable because it is accompanied by additional nutrients and phytochemicals, a fiber supplement should be recommended to close the fiber gap. It has been a continuous effort to improve the physicochemical, functional, sensory, and biological properties of psyllium for promoting its food utilization and enhancing its safety. It is a great challenge to disperse psyllium in water or aqueous solutions even with vigorous agitation because of its extremely strong water-absorbing capacity. The readers are referred to an excellent review focused on the approaches to improve the functionality ( $\mathrm{Yu}$ et al., 2009). Their clinical relevance has been questioned because of the extreme 
unpalatability of products tested with high content of viscose fiber (Ellis et al., 1991). To incorporate lower amounts of fiber into different foods during the day is therefore important to attain the recommended amount for cholesterol lowering (FDA, 1998).

On account of the remarkable property of mucilage from seed husk as a thickener, it could be used as such in food industries. It is employed as a basic stabilizer in ice-creams and as an ingredient of chocolates and other food products. It is also used for sizing purpose and as a base in cosmetics. The husk has been found to be served as a good binder and disintegrated in compressed tablets. On treatment with hot caustic soda solution and subsequent, the seed husk produces jelly which provides a substitute for agar-agar. Isabgol seed gum has been used to prepare dry dentifrice powder and germicidal lubricating gels. It has also been successfully employed in composition for petroleum. Water resistant explosive compositions can be prepared with Isabgol seed gum alone or in admixture with other gums. When mixed with guar, seed husk can be used as some cattle feed particularly in cases of lactating animals. The DE husked seed is around $69 \%$ by weight of the total seed crop which is used as a birdfeed. Psyllium can be used in food and beverages industry, in health drinks, beverages, ice cream, bread, biscuits, other bakery products, rice, cakes, jams, instant noodles, breakfast cereals etc., to improve the fiber content of the food and to increase the bulk of the food. Drinks or flavoured drinks to improve the mouth-feel of the drink and make it richer and impart good consistency to it.

Research indicates that husk is quite safe to use in functional and nutraceutical foods. The FDA has approved the use of food products containing psyllium husk due to its associated health claims (Leeds 2009). Supplementation of fiber, are appreciated by the consumers due to appealing taste and better storage stability. Seeds of psyllium have been used for hundreds of years in traditional Iranian medicinal prescriptions. Because of its pharmacological effects, foods fortified with Plantago ovata mucilage gum may have a superior consumer acceptance. It is commonly found in consumer products such as high fiber breakfast cereals. In addition to being part of fiber formulations, psyllium supplements can also be found in granule, powder, wafer, and capsule forms. And importantly, because psyllium contains an increased amount of soluble fiber gram for gram compared to sources such as oat bran, its use may help fulfill daily dietary fiber recommendations more easily. The effect of soluble fiber from psyllium on body lipids and proteins associated with metabolic process. The functional and nutraceutical foods containing fiber are being developed and studied for their effectiveness with special reference to bakery products, yogurt and drinks (Martin et al., 2008; Perrigue et al., 2009). Bakery products are prepared from different dosage of psyllium husk and literature suggested that replacement of psyllium husk up to $50 \%$ is possible without detrimental change in quality. And also explained the hypocholesterolemic worth of psyllium considering gender and hormonal status in men and pre and postmenopausal women. In postmenopausal women, administrated psyllium (15g/day) for six weeks significantly lowered the total cholesterol concentration $(5.2 \%)$ whereas, in premenopausal women $(1.3 \%)$ whilst, no significant differences observed in triglycerides, apolipoprotein A1 and apolipoprotein B concentration in pre and postmenopausal women. They concluded that postmenopausal women can be benefitted from addition of psyllium husk in their diet for reducing coronary risk (Ganji and Kuo 2008). In other study it was investigated that analyses for fiber added cookies (30\% 
psyllium husk powder) and control (wheat flour) presented the values for carbohydrates as 65.7 and $64.9 \mathrm{~g}$, respectively. While the values for starch, sugar and fiber were 30.3, 20.8 and $12.0 \mathrm{~g}$ in fiber cookies compared with 40.8, 21.0 and only $1.6 \mathrm{~g}$ in control, respectively. There was a marked difference in soluble fiber content as only $0.9 \mathrm{~g}$ in control versus $9.3 \mathrm{~g}$ in psyllium enriched cookies. The protein and fat contents measured were 5.9 and $20.6 \mathrm{~g}$ compared with 4.8 and $20.5 \mathrm{~g}$ in control and psyllium products, respectively while total energy calculated in kJ was 1996 and 1803 in control and fiber cookies, respectively (Vega-Lopez et al., 2001). In processed food to aid weight control, regulation of glucose level for diabetic patients and reducing serum lipid levels in hyperlipidemic due to polysaccharide and its gel-forming nature (Singh 2007). Bioactive profile of different fiber sources is also important like arabinoxylan (AX) that not only improving the quality of baked products but also has pronounced therapeutic potential. Modified samples of the bread dough with psyllium achieved a $93.0 \%$ acceptance rate for individuals with celiac disease and up to $97.0 \%$ for individuals without celiac disease. In terms of chemical composition of the bread dough products had $42.3 \%$ less fat and, consequently, $32.1 \%$ fewer calories. So, psyllium can replace gluten in preparations.

Furthermore, in terms of chemical composition, products made with modified dough had less fat and fewer calories (Zandonadi et al., 2009). The effects of psyllium fiber addition to wheat flour at the levels of $0,1,2,3$ and $4 \%$ on the rheological behavior of the dough. Upon the addition of psyllium fiber, a significant increase in Farinograph (rheological device) water absorption, simultaneously with the increase of the addition level, was recorded. Psyllium fiber added in wheat flour up to $4 \%$ increased the falling number (FN) index. It clearly indicates that incorporating psyllium fiber in wheat flour dough leads to an increase of water absorption. Regarding the falling number index, psyllium fiber addition leads to a decrease of $\alpha$-amylase activity. Therefore, using psyllium fiber at an optimal level, allows an increase of the daily intake of fiber without promoting negative effects on the rheological properties of the dough (Mironeasa et al., 2013). In a studied the development of "Spongy Dessert" by incorporating the "mucilage" powder extracted from the seeds of psyllium (Plantago ovata). The mucilage powder was incorporated at 3.0, $3.54 .0 \& 4.5 \%$ level in the Chhena (milk solid) prepared from the low fat cow milk.

On the basis of results obtained through organoleptic evaluation, the Spongy Dessert prepared by incorporating $4 \%$ mucilage was selected and analyzed for nutrients. The developed product namely herbal rassogolla prepared by incorporating $4.0 \%$ mucilage powder was a good source of protein (13.8 $\mathrm{g} / 100 \mathrm{~g}$ ) and dietary fibre (1.5), low in carbohydrates $(18.8 \mathrm{~g} / 100 \mathrm{~g})$, saturated fat $(0.8$ $\mathrm{g} / 100 \mathrm{~g})$ and energy $(141.2 \mathrm{Kcal})$ and free from trans-fat. This mucilaginous spongy dessert has the properties to provide relief from constipation and acidity (Garg et al., 2014). Gums and Mucilage are naturally occurring biopolymers, finding increasing applications in pharmaceutical and biotechnology industry. It has been used successfully for many years in the food and pharmaceutical industry as a thickening agent, as a gelling agent, and as a colloidal stabilizer. Mucilage also has several unique properties that have enabled it to be used as a matrix for entrapment and/or delivery of variety of drugs, proteins, and cells. Being a naturally occurring polysaccharide, in recent year it has gained increased importance in industrial applications (Majmudar et al., 2002). 
Int.J.Curr.Microbiol.App.Sci (2020) 9(12): 1949-1959

Fig.1 Steps involved in manufacturing of psyllium husk

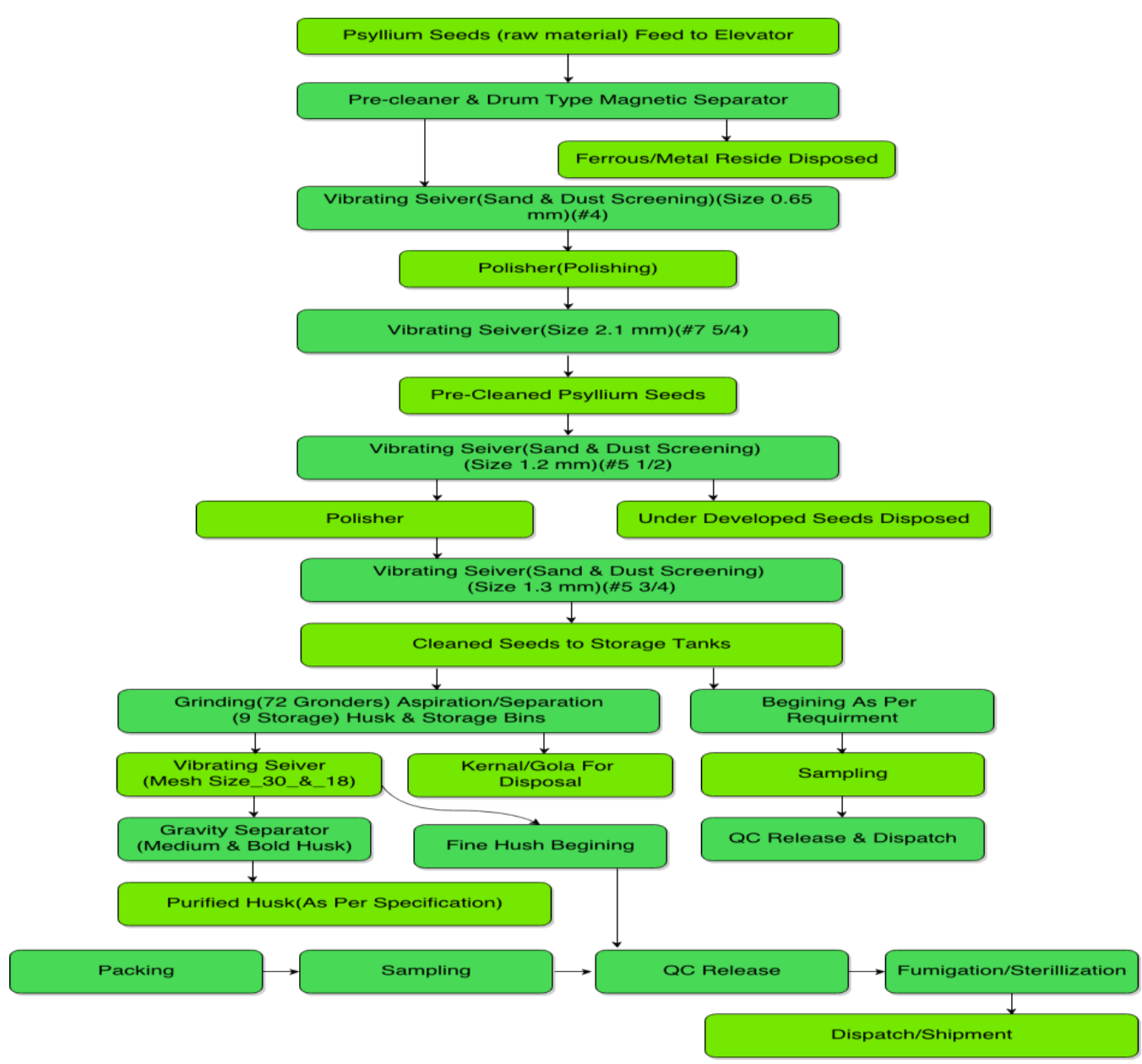

Fig.2 Therapeutic benefits of psyllium husk

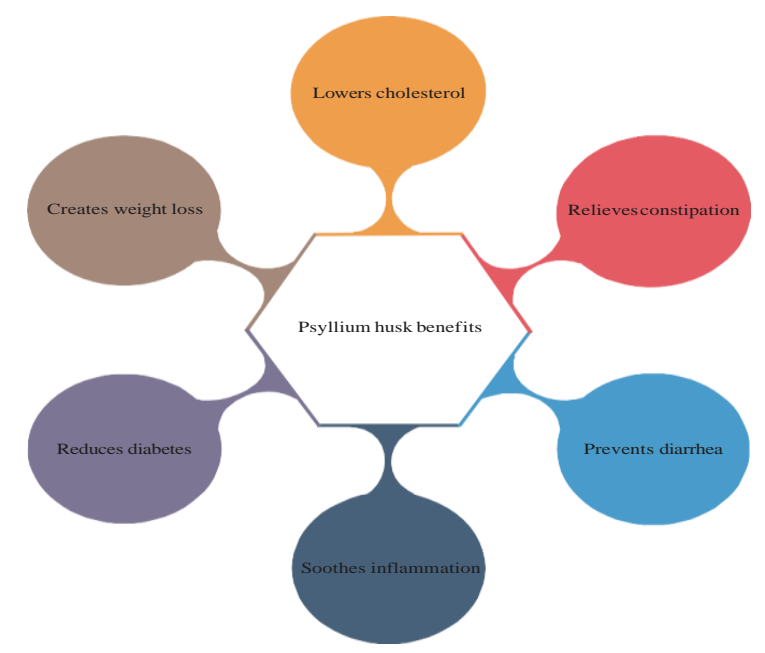


Prebiotics are food ingredients that stimulate selectively the growth and activity of bifidobacteria and lactobacilli in the gut and thereby benefit health (Cummings and Macfarlane, 2002). The defining property of prebiotics is their effect on the microflora of the large bowel (Kanauchi et al., 2003; Rodriguez-Cabezas et al., 2003). Poor microbiologic evidences are nevertheless available concerning the ability of Psyllium derivates to promote the growth of bifidobacteria in human gut and thus act as a prebiotic. A recent study suggests that Psyllium seed husk can be metabolized by bifidobacteria only after partial hydrolysis (Elli et al., 2008). This study in women demonstrates that the bifidogenic potential can only be detected in healthy subjects with low levels of fecal bifidobacteria before Psyllium supplementation.

\section{Therapeutic benefits of psyllium husk}

Psyllium has been reported for the treatment of constipation, diarrhea, and irritable bowel syndrome, inflammatory bowel diseaseulcerative colitis, colon cancer, diabetes and hypercholesterolemia (Fig. 2).

\section{Constipation and hemorrhoids}

Psyllium has been shown to have the paradoxical property of both improving constipation by increasing stool weight and ameliorating chronic diarrhea. Several studies suggest that psyllium may provide benefits for treating constipation. There is a scientific basis for psyllium working as a mild laxative. Water absorbed by psyllium husk is not absorbed by the intestine resulti.ng in making stools softer and bulkier leading to a smooth defecation process. In hemorrhoids veins in the walls of the anus or rectum are dilated, which is usually caused by untreated constipation but sometimes associated with chronic diarrhea. Adequate fiber supplement combined with the TONE method can stop the progression of hemorrhoids and bleeding, and preventing surgery in most patients with advanced hemorrhoids.

\section{Diarrhea}

The normal intestine delivers stools that defer widely in quantity but maintain percent fecal water within a narrower range. Stools looseness in diarrhea is determined by the ratio of fecal water too water holding capacity of insoluble solids. Psyllium increases the number of normal stools and decreases the number of liquid stools. A combination of psyllium and calcium seems to be cheap and effective alternative to conventional treatment of chronic diarrhea. Fecal consistency was markedly different in psyllium calves as compare with control.

\section{Ulcerative colitis (Crohn's disease)}

The two primary sites for Crohn's disease are the ileum, which is the last portion of the small bowel (ileitis, regional enteritis), and the colon (Crohn's colitis). A small number of studies have examined the ability of psyllium to maintain remission in ulcerative colitis. Dietary fiber has been proven to be beneficial in maintaining remission in human ulcerative colitis, an effect related with an increased Luminal production of short-chain fatty acids (SCFA). Dietary fiber Supplementation ameliorated colonic damage in HLA-B27

\section{Irritable bowel syndrome}

Constipation is defined as a symptom chronic constipation -based disorder, for at least 3 months in a year for the unsatisfactory defecation and characterized by infrequent stools, difficult stool passage, or both. On the other hand, the presence of clinically 
important abdominal discomfort or pain associated with constipation defines IBS with constipation. Intake of psyllium may be effective in alleviating chronic constipation in patients without slow colonic transit or disordered constipation. On the other hand, fiber with lactulose may improve stool consistency in patients with IBS with constipation. Personality factors influence the magnitude of therapeutic response of the psyllium. The easing of bowel dissatisfaction appears to be a major reason for the therapeutic success of psyllium in IBS.

\section{Diabetes}

Psyllium has been proposed as a possible treatment for high blood sugar levels. Psyllium is a soluble fiber which forms gel in the intestine which leads to slowing the rate of nutrient absorption and results in lowering postprandial blood glucose level. Studies in humans suggest moderate reductions in blood sugar levels after a single dose of psyllium, with unclear long-term effects. Water- soluble dietary fibers decrease postprandial glucose concentrations and decrease serum cholesterol concentrations to men with type 2 diabetes. Early or uncontrolled studies suggested that psyllium improved glycemic and lipid control in individuals with type- 2 diabetes. The ability of soluble fibers to reduce the postprandial glucose response to meals eaten several hours after fiber ingestion (second meal effect) was shown previously in nondiabetic individuals

\section{Cholesterol lowering}

It has been observed that there is a positive association with plasma LDL cholesterol levels and coronary heart disease risk. Intake of dietary fibers known to lower the concentration of LDL in plasma is considered to be highly beneficial. Psyllium intake has consistently shown significant reductions in plasma LDL cholesterol levels ranging from 10 to $24 \%$. Reports of the use of psyllium, largely in hypercholesterolemia men, have suggested that it lowers serum cholesterol as a result of the binding of bile acids in the intestinal lumen and reduced risk of coronary heart disease. The mechanism of action of psyllium's hypercholesterolemia effects has not been fully elucidated. Psyllium was shown to stimulate bile acid synthesis by increasing the hydroxylase activity in animal and humans' models.

\section{Gastro Esophageal Reflux Disease (GERD)}

GERD, is a digestive disorder that affects the lower esophageal sphincter (LES), it has been shown that frequency of symptoms in patients who suffer periodically from Gastro esophageal reflux disease (GERD) with constipation was reduced by the intake of psyllium as compared with omeprazole.

\section{Hypertension}

Psyllium, soluble fiber is part of a healthy diet and exerts a protective effect in normalizing the blood pressure. Observational studies show that when fiber is increased in diet (by $7-15 \mathrm{~g} /$ day above the usual levels) is significantly associated with reduced blood pressure and hypertension risk compared to the low fiber intake in Western diets. Consumption of soluble fiber is proved to reduce overall Systolic Blood Pressure (SBP) and diastolic blood pressure (DBP) in all populations, and especially psyllium fiber helps to reduce overall SBP.

In conclusion the increased fiber intake is consistently associated with better health, reduced chronic disease risk, and healthy aging. The utilization of psyllium husk is limited to treat constipation, diarrhea and hypercholesterolemia. There are many hidden benefits of Psyllium husk, if taken in an 
appropriate amount according to RDA it can be a part of our regular diet. While consuming psyllium, water intake should be increased, in order to take full advantage. People are unaware of its benefits in treatment of diabetes, weight loss, obesity, high blood pressure and heart disease. Though much researches have been conducted to investigate the health promoting effects of psyllium husk, a very few studies have been done on developing food products fortified with psyllium. Therefore, there is a need to elaborate health benefits of psyllium husk in terms of its nutritional quality to emanate the public health disorders and make food products at household level.

\section{References}

Al-Assaf, S., Phillips, G.O., Williams, P.A., Takigami, S., Dettmar, P. and Havler, M. Molecular weight, tertiary structure, water binding and colon behaviour of ispaghula husk fibre. Proceedings of the Nutrition Society. 62: 211-21(2003).

Anderson JW, Jones AE, Riddell-Mason S. Ten different dietary fibers have significantly different effects on serum and liver lipids of cholesterol-fed rats. J. Nutr. 124,1994,78-83.

Askari, H., Farahnaki, A., Majzoobi, M. and Mesbahi, G.H. Hydrocolloid extraction from Psyllium husk and investigation on its rheological properties. 18th, National Iranian food science and technology conference.

Bijkerk CJ, Muris JW, Knottnerus, JA, Hoes AW, de Wit NJ, Systematic review: The roll of different types of fibre in the treatment of irritated bowel syndrome. Aliment. Pharmacom. Ther. 19, 2004, pp.245-251.

Bouchoucha MG, Faye A, Savarieau B, ArsacM, Effect of an oral bulking agent and a rectal laxative administered alone or in combination for the treatment of constipation. Gastroenterol. Clin.Biol.28, 2004, pp.438-443.

Brad JC, Calagiuri S, Crossman S, Allen A, Robertes DC, Truswell AS, Lowglycemic index foods improve longterm glycemic control in NIDDM. Diabetes Care 14, 1995, pp.95-101.

Brennan CS, Dietary fiber, glycemic response, and diabetes. Mol. Nutr. Food Res.49, 2005, pp.560-570.

Burton R, Manninen V, Influence of a psyllium-based fibre preparation on faecal and serum parameters. Acta Med. Scand.Suppl.668, 1982, pp.9194.

Clausen MR, Bonnen H, Mortensen PB, Colonic fermentation of dietary fiber to short chain fatty acids in patients with adenomotous polyps and colonic cancer. Gut 32, 1991, pp.923- 928.

Cummings, J.H., Edmond, L.M. and Magee, E.A. 2004. Dietary carbohydrates and health: Do we still need the fiber concept? Clinc. Nutr. Suppl. 1:5-17 (2008).

Degan LP, Phillips SF, how well dose stool form reflects colonic transit? Gut 39, 1996, pp.109-113.

Farahnaki, A., Askari, H., Majzoobi, M. and Mesbahi, G.H. The impact of concentration, temperature and $\mathrm{pH}$ on dynamic rheology of Psyllium gels. $J$. of Food Engineering.1-8 (2010).

Fischer, H.M., Nanxiong, Y., Ralph, R.G.J., Andersond, L. and Marletta, J.A. The gel forming polysaccharide of psyllium husk (Plantago ovata Forsk). Carbohydrate Research. 339:20092017 (2004).

Ganji, V. and Kuo, J. Serum lipid responses to psyllium fiber: differences between pre- and post-menopausal, hypercholesterolemic women. Nutrition Journal, 7:22 
doi:10.1186/1475-2891-7-22 (2008).

Garg, P., Raghav, P.K., Sharma, R.K., Jasuja, N.D., Sharma, R., and Agarwal, N. Development of Mucilaginous Spongy Dessert-A herbal rassogolla prepared from cow milk. International J. of Scientific and Research Publications. 4(2) ISSN 2250- 3153(2014).

Galisteoa, M., Morona, R., Riveraa, L., Romeroa, R., Anguerab, A. and Zarzueloa, A. Plantago ovata huskssupplemented diet ameliorates metabolic alterations in obese Zucker rats through activation of AMPactivated protein kinase. Comparative study with other dietary fibers. Clin. Nutri. 29:261-267 (2010).

Guo, Q., Cui, S.W., Wang, Q. and Young, J.C. Fractionation and physicochemical characterization of psyllium gum. Carbohyd. Polym. 73:35-43 (2008).

Koch A, Voderholzer WA, Klauser AG, Muller-LissnerS, Symptoms in chronic constipation. Dis. Colon Return 40, 1997, pp.902-906.

Krammer H., Schieger F, Singer MV., Therapeutic option of chronic constipation. Internist (Berl.) 46, 2005, pp.1331-1338.

Kumar A, Kumar N, Vij JC, Sarin SK, Anand BS, Optimal dosage of ispaghula husk in patients with irritable bowel syndrome: correlation of symptom relief with whole gut transit time and stool weight. Gut 28, 1987, pp150-155.

Leeds, A.R. Dietary Fiber; Role in Nutrition Management of Disease. In: Guide to Nutritional Supplements. Caballero, B. (ed.). Academic press, USA (2009).

Li, L., Tsao, R., liu, S., Yang, J. C., Zhu, H., deng, Z., Xie, M. and Fu, Z. Isolation and purification of acetoside and isoacetoside from Plantago Psyllium L. by high speed counter current chromatography. $\quad J . \quad$ of
Chromatography.

1063:161-169 (2005).

Majmudar, H.; Mourya, V.; Devdhe, S. and Chandak, R. Pharmaceutical Applications of Ispaghula Husk: Mucilage. International J. of Pharmaceutical Sciences Review \& Research. 18(1):49-55 (2002).

Martin-Diana, A.B., Rico, D. and BarryRyan, C. Green tea extract as a natural antioxidant to extend the shelf- life of fresh-cut Lettuce. Innovative Food Sci. Emerging Technol. 9:593-603 (2008).

Mironeasa, S., Codină, G.G. and Popa, C. Effect of the addition of Psyllium fiber on wheat flour dough rheological properties. Recent Researches in Medicine, Biology and Bioscience. ISBN: 978-960-474-326-1 (2013).

Moreno, L.A., Tresaco, B., Bueno, G., Fleta, J., Rodriguez, G., Garagorri, J.M. and Bueno, M. Psyllium fiber and the metabolic control of obese children and adolescents. J. Physiol. Biochem. 59:235-242 (2003).

Pal, S., Khossousi, A., Binns, C., Dhaliwal, S. and Radavelli-Bagatini, S. The effects of 12-week psyllium fiber supplementation or healthy diet on blood pressure and arterial stiffness in overweight and obese individuals. $\mathrm{Br}$. J. Nutr. 26:1-10 (2011).

Perrigue, M.M., Monsivais, P. and Drewnowski, A. Added soluble fiber enhances the satiating power of lowenergy-density liquid yogurts. J. Am. Diet. Assoc. 109:1862-1868 (2009).

Qvitzau S, Matzen P, Madsen P, Treatment of chronic diarrhoea: loperamide versus ispaghula husk and calcium. Scand. J. Gastroenterol. 23, 1988, pp.12371240.

Romero-Baranzini, A. L , Rodriguez, O. G., Yanez- Farias, G. A., Barron-Hoyos, J. M. and Rayas-Duarte, P. Chemical, Physicochemical, and Nutritional 
Evaluation of Plantago (Plantago ovata Forsk). Cereal chem. J. 83(4): 358-362 (2006.

Singh, B. Psyllium as therapeutic and drug delivery agent. Int. J. Pharmaceutics. 334: 1-14 (2007).

Theuissen, E. A. M. Water soluble dietary fibers and cardiovascular disease. Physiology and Behavior. 94: 285-292 (2008).

Thompson, WG, Longstreth, GF, Drossman, DA, Heaton, KW, Irvinej, MullerLissner, SA, 1999. Functional bowel disorders and functional abdominal pain. Gut 45, pp. II43-II47.

Van-Craeyveld, V.V., Delcour, J.A. and Courtin, C.M. Extractability and chemical and enzymic degradation of psyllium (Plantago ovata Forsk) seed husk arabinoxylans. Food Chem. 112:812-819 (2009).

Vega-Lopez, S., Vidal-Quintanar, R.L. and Fernandez, M.L. Sex and hormonal status influence plasma lipid responses to psyllium. Am. J. Clin. Nutr. 74:435-441 (2001).

Vyth, E.L., Steenhuis, I.H.M., Roodenberg, A.J.C., Brug, J. and Seidell, J.C. Frontof-pack nutrition label stimulates healthier product development; a quantitative analysis. Intl. $J$. Behavioral Nutr. Physical Activity. 7:65 (2010).

Yu, L., Lutterodt, H. and Cheng, Z. Beneficial health properties of psyllium and approaches to improve its functionality. In: Advances in Food and Nutrition Research. Taylor, S. (ed.). 55:193-217 (2009).

Zandonadi, R.P., Botelho, R.B. and Araújo, W.M. Psyllium as a substitute for gluten in bread. J. of the American Dietetic Association. 109(10):1781-4 (2009).

\section{How to cite this article:}

Katke, S. D., H. W. Deshpande and Tapre, A. R. 2020. Review on Psyllium Husk (Plantago ovata): A Novel Superfood for Human Health. Int.J.Curr.Microbiol.App.Sci. 9(12): 1949-1959. doi: https://doi.org/10.20546/ijcmas.2020.912.232 